\title{
Prévention du VIH: importance primordiale d'un conseil médical de qualité
}

\author{
Article Meystre-Agustoni: commentaires de I'Office fédéral de la santé publique (OFSP) et de la Commission fédérale \\ pour les problèmes liés au sida (CFS)
}

Office fédéral de la santé publique, Division Maladies transmissibles, Section Sida

En Suisse, la prévention du VIH repose sur trois niveaux d'intervention: l'information destinée à la population (campagne STOP SIDA), l'information interpellant des groupes cibles spécifiques et le conseil personnalisé. La plupart du temps, le conseil personnalisé est dispensé au cabinet médical, mais le potentiel que représente cette rencontre n'est pas encore assez exploité. En vue de soutenir les médecins, la Commission d'experts clinique et thérapie VIH/sida (CCT) a élaboré des recommandations sur les conseils en matière de test VIH («Guide» dans cette édition).

a L'article se concentre sur le conseil dispensé à des personnes séronégatives ou au statut sérologique inconnu; le conseil préventif des séropositifs n'y est pas abordé, mais représente un thème que l'OFSP continuera de développer dans le programme national VIH et sida 2004-2008.

b Selon l'enquête suisse sur la santé et les données de l'OFSP.

c Cf. notamment les publications suivantes: [2-4].
Le présent article de Meystre-Agustoni et al. est consacré à l'anamnèse du risque et au conseil sur le VIH dans les cabinets médicaux suisses ${ }^{\mathrm{a}}$. Il montre que la situation prévalant depuis 1990 est relativement bonne mais susceptible d'amélioration.

Il est réjouissant de constater que l'anamnèse du risque VIH n'est pas menée uniquement dans les groupes habituellement considérés à risque. Toutefois le VIH n'est pas encore assez thématisé dans certains groupes (p.ex. les migrants), et il est même moins abordé qu'auparavant au sein du groupe à risque «classique» des hommes homosexuels.

Indépendamment du test VIH, le conseil préventif individualisé peut être pertinent et important, notamment en cas de questions sur les règles du safer sex ou sur l'intégration de la prévention dans une situation personnelle. Mais le conseil préventif reste souvent lié au test VIH qui est très demandé par la population suisse: selon une étude récente [1] réalisée auprès d'un échantillon aléatoire, $41 \%$ des personnes entre 18 et 45 ans ont effectué un test VIH au moins une fois, test effectué lors de dons du sang non compris. En Suisse, on compte chaque année 300000 tests VIH hors don du sang ${ }^{\mathrm{b}}$. Par conséquent, le conseil individualisé dans le cadre du test VIH représente un atout majeur de la prévention dans ce domaine.

En Suisse, près de la moitié (48\%) des tests VIH présentant un résultat positif sont effectués dans des cabinets médicaux. Les médecins occupent donc une position privilégiée en matière de conseil individualisé. D'après la présente étude, ils sont conscients de l'importance de leur rôle.

S'agissant du test VIH, le modèle du VCT (Voluntary Counseling and Testing ou conseil et dépistage volontaire) fait office de procédure standard. Développé au milieu des années 80 par ONUSIDA, il est destiné à tous ceux qui souhaitent connaître leur statut infectieux et effectuer un test VIH. Les principaux éléments de ce modèle sont fixés dans les recommandations de plusieurs organisations de santéc. Depuis quelques temps, les Etats-Unis encouragent le test VIH à large échelle, mais il est difficile de savoir à quel point le conseil qui l'accompagne est assuré; la question se pose notamment en ce qui concerne le test VIH effectué sur un prélèvement de salive qui est maintenant autorisé aux Etats-Unis. Dans ce contexte, la garantie de la qualité du conseil reste plus que jamais d'actualité.

Le présent article et des études menées auprès des utilisateurs de l'offre montrent que le potentiel de conseil dispensé dans le cadre du test VIH n'est que partiellement utilisé en Suisse: ainsi, $44 \%$ des hommes homosexuels qui ont effectué un test VIH en 2002 n'ont reçu de conseil ni avant ni après le test [5]. D'après la présente étude, lors des consultations de test, un tiers des médecins n'abordent pas toujours la question du délai entre l'exposition au virus et le moment à partir duquel on peut le détecter, et plus de la moitié d'entre eux ne cherchent pas systématiquement à identifier le risque. Cette constatation est d'autant plus inquiétante qu'elle remet en question non seulement l'utilité du test en tant qu'outil de prévention, mais également la fiabilité du résultat.

Les résultats de Meystre-Agustoni et al. sont complétés par une étude linguistique [6], récemment parue, effectuée auprès de 620 médecins travaillant en Suisse romande. La plupart d'entre eux indiquent que leur jargon médical habituellement utilisé est plutôt défavorable pour aborder des thèmes tels que la sexualité et le VIH/sida avec leurs patients. Il existe également, des deux 
côtés, une certaine gêne à évoquer le sujet, et les médecins manquent souvent de temps pour le conseil VIH. Près de la moitié reconnaît l'importance d'une formation supplémentaire afin de bien maîtriser la communication non verbale ainsi que les techniques d'entretien et de conseil.

L'OFSP et la CFS reconnaissent que le soutien des médecins, pour permettre une anamnèse du VIH et un conseil de haute qualité, peut être amélioré. Il existe bien des informations détaillées à l'attention des médecins sur le dépistage et le diagnostique [7], de même que des recom- mandations sur le test VIH pendant la grossesse [8] et un concept de test VIH 2004 révisé [9]. Mais une notice succincte relative à l'anamnèse du risque et au conseil et dépistage du VIH fait actuellement défaut. Dans le domaine des laboratoires, du diagnostic et de la thérapie du VIH/ sida, des recommandations de ce type sont des instruments bien établis visant à soutenir le personnel médical. Pour pallier cette situation, la CCT publie dans le présent bulletin des recommandations pour les conseils en matière de test VIH («Guide»).

\section{Messages de prévention}

(d'après le Programme national VIH et sida 2004-2008, p. 25)

- Chaque personne doit veiller elle-même, dans le cadre de ses possibilités, à se protéger d'une infection par le VIH.

- Toute personne sexuellement active qui ne vit pas dans une relation de fidélité réciproque et exempte de risque d'infection au VIH doit observer les règles du safer sex:

- toujours utiliser un préservatif de bonne qualité en cas de rapports avec pénétration;

- ne jamais prendre en bouche ni avaler de sperme ou de sang menstruel.

- Toute personne sexuellement active qui ne vit pas dans une relation de fidélité réciproque et exempte de risque d'infection au VIH doit s'informer des risques et des possibilités de protection et se familiariser avec l'usage du préservatif.

- Dans une relation de fidélité réciproque, les partenaires ne devront renoncer à l'utilisation de préservatifs que trois mois, au plus tôt, après avoir bénéficié, en commun, d'un conseil et s'être soumis à un test VIH jugé négatif.

- Un test VIH ne protège pas du sida. Quiconque pense cependant avoir eu un comportement à risque peut s'informer sur sa situation en se soumettant à un test, ce qui lui permettra éventuellement de profiter d'un traitement précoce de l'infection au VIH.

- Quiconque s'injecte des drogues doit toujours utiliser une seringue neuve et du matériel propre (cuillère, filtre, ouate, eau) et ne pas les partager avec d'autres consommateurs (safer use).

- Le VIH et le sida peuvent faire l'objet d'un traitement, mais ne sont pas curables. Le traitement est compliqué, difficile, coûteux et incertain quant à son résultat. Le préservatif reste la meilleure protection contre le $\mathrm{VIH} /$ sida.

- Le VIH/sida est, et demeure, un problème de société. Le soutien et la solidarité à l'égard des personnes touchées et menacées sont une nécessité.

- La prévention du VIH a des limites. La compréhension et l'ouverture vis-à-vis des personnes atteintes du VIH/sida sont les constituants d'une société solidaire et renforcent, à leur tour, la prévention du VIH.

- Le VIH ne se transmet pas par les baisers, par des contacts sociaux comme des poignées de main, des embrassades ou des caresses, par des éternuements ou une expectoration, par des repas et boissons pris en commun ou par l'utilisation des mêmes assiettes, verres et couverts, par le travail ou par le partage d'un logement avec des personnes vivant avec le VIH/sida, par l'utilisation des mêmes salles de bain, toilettes ou saunas, pas plus que par des piqûres d'insectes.

\section{Références}

1 Renzi C, Zantedeschi E, Signorelli C, NEM Group. Voluntary HIV testing in Europe. Scand J Public Health 2004;32:102-10.

2 WHO. Increasing access to knowledge of HIV status: conclusions of a WHO consultation, 3-4 December 2001. Geneva: WHO, 2002.
3 UNAIDS. Voluntary Counselling and Testing: a gateway to prevention and care. UNAIDS Case study. Geneva: UNAIDS; 2002.

4 CDC. Revised Guidelines for HIV Counseling, Testing, and Referral (CTR) and Revised Recommendations for HIV Screening of Pregnant Women. MMWR 2001;50:1-58. 
5 Häusermann M, Wang J. projet santé gaie. Les premiers résultats de l'enquête sur la santé des hommes gais de Genève. Genève: Dialogai; 2003. www.dialogai.org/pdfs/brochurecomplet.pdf.

6 Singy P. Le sida au cabinet médical. Les mots pour en parler. Genève: Editions Médecine et Hygiène; 2004.

7 Office fédéral de la santé publique. VIH - état actuel des connaissances sur le dépistage et le diagnostic. Informations à l'intention des médecins. Berne: Office fédéral de la santé publique (OFSP) en collaboration avec la Commission fédérale pour les problèmes liés au sida (CFS), 2000. www.suchtundaids.bag.admin.ch/themen/aids/ doku/publikationen/00972/index.html? language $=$ fr $\&$ dir $2=\&$ schriftgrad $=$.
8 Commission d'experts clinique et thérapie VIH/sida. Test VIH pendant la grossesse. Recommandations de la Commission d'experts clinique et thérapie VIH et sida (FKT). Bulletin de l'office fédéral de la santé publique 2003;9:152-3. www.suchtundaids.bag.admin.ch/imperia/md/ content/aids/19.pdf.

9 Commission laboratoire et diagnostic. Concept de test VIH 2004: nouvelles directives dans le domaine du dépistage. Bulletin de l'office fédéral de la santé publique 2004;10:168-70. www.suchtundaids.bag.admin.ch/imperia/md/ content/aids/hiv-testkonzept_2004_f.pdf.

\section{Entretien pré- et post-test VIH: petit guide pour les cabinets médicaux}

Pietro Vernazza, Commission d'experts clinique et thérapie VIH/sida

En Suisse, les cabinets médicaux et les hôpitaux effectuent environ 300000 tests VIH par an. Un bref entretien doit accompagner chaque test. Le présent guide réunit les points essentiels pour réaliser cet entretien.

\section{Principes du test VIH}

- Les personnes qui passent souvent un test pour savoir si elles ont une infection au VIH ont, en général, un comportement à risque répété.

- Chaque test VIH offre la possibilité de bénéficier d'un bref entretien de prévention.

- Un test VIH sans entretien est une occasion manquée pour bénéficier de la prévention en la matière.

- Le travail de prévention fait partie des tâches essentielles du médecin de premier recours.

\section{Entrées en matière possibles pour l'entretien pré-test}

\section{Questions sur les comportements à risque}

- Quand auriez-vous pu dernièrement contracter une infection par le VIH?
- Comment pouvez-vous être si sûr que vous n'auriez pas pu être infecté après?

- D'après vous, combien de temps faut-il pour qu'un test se révèle positif?

- Depuis le contact à risque, avez-vous eu de la fièvre, une éruption cutanée sur le corps ou d'autres symptômes?

Questions sur les comportements préventifs

- Que savez-vous des moyens de protection possibles?

- Quelles sont, d'après vous, les plus grandes difficultés à surmonter quand il s'agit de prévention du VIH?

- D'après vous, la prévention du VIH est-elle plus importante que la prévention d'autres infections sexuellement transmissibles?

- Avez-vous déjà eu des problèmes lors de l'utilisation d'un préservatif?

- Savez-vous comment utiliser un préservatif? Pourriez-vous l'expliquer à quelqu'un d'autre?

\section{Le test est négatif: fausses interprétations fréquentes}

- Alors je peux être certain que le/la partenaire de mon dernier contact sexuel est aussi 
négatif, même si je ne connais pas son statut sérologique:

$\rightarrow$ Faux: le partenaire peut très bien être séropositif. Il n'y a simplement pas encore eu infection.

- Alors j'ai agi comme il fallait jusqu'ici et je peux continuer ainsi:

$\rightarrow$ Faux: le comportement adopté jusqu'à présent peut tout à fait comporter un risque. Il n'a tout simplement pas encore conduit à une infection.

- Alors j'ai eu de la chance la semaine passée: $\rightarrow$ Faux: ce n'est qu'au bout de trois mois au plus tôt que l'on pourra exclure avec certitude toute infection à la suite de cette exposition au risque de la semaine passée.

- Alors je ne dois rien dire à mon partenaire:

$\rightarrow$ Faux: étant donné que le moment de l'exposition au risque ne remonte pas encore à trois mois, une infection ne peut être exclue avec certitude. Le/la partenaire reste par conséquent exposé/e.

- Alors les symptômes que j'ai développés ne sont pas dus à une infection au VIH:

$\rightarrow$ Attention: en cas de soupçon de primoinfection au VIH, le virus du VIH doit absolument être détecté (par un seul test); une deuxième recherche du virus et des anticorps (test combiné) dans les 5 à 8 jours est encore plus précise.

\section{Informations essentielles avant de réaliser un test rapide}

- Le test rapide du VIH ne peut pas révéler une infection aiguë (pas d'antigènes du VIH). Un test anticorps/antigènes combiné ( $4^{\mathrm{e}}$ génération) est plus approprié.

- Les tests rapides du VIH sont des tests de recherche. Ils peuvent exclure efficacement une infection au VIH au plus tôt après 3 mois. Un test positif peut tout à fait s'avérer faussement positif (environ 1 pour 1000 tests négatifs). Si la probabilité pré-test est basse, la valeur prédictive positive peut se situer entre 50 et $70 \%$. Pour exclure toute infection, le sang doit être envoyé dans un laboratoire. Il est recommandé d'en informer les personnes avant qu'elles effectuent le test rapide.

\section{Références}

- Office fédéral de la santé publique. VIH - état actuel des connaissances sur le dépistage et le diagnostic. Informations à l'intention des médecins. Berne: Office fédéral de la santé publique (OFSP) en collaboration avec la Commission fédérale pour les problèmes liés au sida (CFS); 2000. www.suchtundaids.bag.admin.ch/themen/aids/ doku/publikationen/00972/index.html?language= fr\&dir $2=\&$ schriftgrad $=$.

- Commission laboratoire et diagnostic. Concept de test VIH 2004: nouvelles directives dans le domaine du dépistage. Bulletin de l'office fédéral de la santé publique 2004;10:168-70. www.suchtundaids. bag.admin.ch/imperia/md/content/aids/hivtestkonzept_2004_f.pdf. 